\title{
Mobile Evaluation of Heart Rate Variability Using the Diver's Reflex
}

\author{
Harrison Seltzer, Melissa Pellman, Raechel Warchock, Joseph Billian, and Robert Baker \\ Western Michigan University, Homer Stryker M.D. School of Medicine, Kalamazoo, Michigan, USA
}

\begin{abstract}
Introduction. Heart rate variability (HRV) is considered a marker of autonomic nervous system (ANS) function and a biomarker of interest in evaluating nervous system function following traumatic brain injury. This study validates prior research with larger sample sizes and proposes a model for establishing baseline HRV reactivity in healthy participants. Methods. Sixty-two healthy collegiate athletes were recruited for this study. Following informed consent, they were evaluated supine using the Elite HRV CorSense monitor and platform to record low frequency/high frequency (LF/HF) ratio and root means square of successive differences (RMSSD) over 5 min. A bag of ice was placed on their face, then RMSSD and LF/HF ratio were collected over three successive 1-min intervals. Results. RMSSD was elevated at 1 and $2 \mathrm{~min}(+47.4 \mathrm{~ms}, p<.0001 ;+16.5 \mathrm{~ms}, p=.014)$ following face cooling and fell to baseline at $3 \mathrm{~min}(+4.6 \mathrm{~ms}, p=.52)$. LF/HF ratio decreased following face cooling at 2 and 3 min (change from rest \%: $2 \mathrm{~min},-33 \%, p=.007 ; 3 \mathrm{~min},-50 \%, p=<.0001$ ). Conclusion. The Elite HRV platform can detect an elevation in RMSSD in the first minute following face cooling with a return to baseline in the second and third minutes. It can also detect a consistent decrease in LF/HF following face cooling.
\end{abstract}

Keywords: HRV; RMSSD; LF/HF; concussion; CorSense

Citation: Seltzer, H., Pellman, M., Warchock, R., Billian, J., \& Baker, R. (2021). Mobile evaluation of heart rate variability using the Diver's Reflex. NeuroRegulation, 8(2), 96-103. https://doi.org/10.15540/nr.8.2.96

*Address correspondence to: Harrison Seltzer, BS, 637 Denner

Street, Kalamazoo, MI 49006, USA. Email: harrison.seltzer@med.wmich.edu

Copyright: $\odot$ 2021. Seltzer et al. This is an Open Access article distributed under the terms of the Creative Commons Attribution License (CC-BY).
Edited by:

Rex L. Cannon, PhD, SPESA Research Institute, Knoxville, Tennessee, USA

\section{Reviewed by:}

Cathlyn Niranjana Bennett, PhD, Christ University, Bangalore, Bengaluru, India

Wajida Perveen, Sialkot College of Physical Therapy, Sialkot, Pakistan

\section{Introduction}

Heart rate variability (HRV) is defined as the fluctuation in time intervals per heartbeat (Shaffer \& Ginsberg, 2017). HRV that is too low reflects impaired adaptability of the cardiorespiratory system to stress, whereas HRV that is too high can be indicative of pathologic states which can confer an elevated risk of mortality (Stein et al., 2005). Clinically HRV has been used to evaluate patients with serious arrhythmias or other high mortality cardiac conditions to predict cardiac function in conjunction with other clinical markers (Kleiger et al., 2005). Of particular interest to sports medicine is HRV's role as a marker of autonomic dysfunction in cases of concussion, as research is demonstrating HRV could be used to quantitively evaluate autonomic recovery from brain injury (Gall et al.,
2004; Katz-Leurer et al., 2010; Senthinathan et al., 2017; Vistisen et al., 2014).

The term "HRV" refers to a set of data acquired from a reading that includes RR-intervals, root means square of successive differences (RMSSD), low frequency/high frequency (LF/HF) ratio, and many others (Shaffer \& Ginsberg, 2017). RMSSD and the LF/HF ratio have been shown to be different between healthy and postconcussion subjects and so are the focus of this study (Johnson et al., 2018; Senthinathan et al., 2017). RMSSD reflects the individual beat-to-beat variances in heart rate over a measured interval and has been shown to estimate autonomic nervous system (ANS) effects on HRV (Shaffer \& Ginsberg, 2017). The LF/HF ratio measures the relative power of the LF and HF bands in spectral analysis of HRV (Shaffer \& Ginsberg, 2017). The LF portion $(0.04-0.15 \mathrm{~Hz})$ is controlled 
by both the sympathetic (SNS) and parasympathetic nervous system (PNS), while the HF portion (0.15$0.40 \mathrm{~Hz}$ ) is predominantly controlled by the PNS alone (Shaffer \& Ginsberg, 2017).

Following a concussion, the decision to return to play is a complicated one made jointly by athletes, coaches, and clinicians. Current guidelines suggest return to play is appropriate when symptom scores, neurologic evaluation, and cognitive activity all return to baseline (Patricios et al., 2018). Most adolescent and collegiate athletes recover from an uncomplicated concussion in 7 to 10 days (Leddy et al., 2012). Unfortunately, for some athletes, symptoms may persist after the return to play decision is made and can possibly progress into postconcussive syndrome where symptoms prevent the athlete from playing for months after their injury (Miranda et al., 2018). Autonomic and vestibular deficits in particular have been shown to persist in some athletes even after return to play decisions have been made (Blake et al., 2016; Collins et al., 2016; Esterov \& Greenwald, 2017). Because HRV is a reflection of the ANS it can be used as an objective marker to measure physiologic recovery especially when there is a baseline prior to injury for comparison.

One limitation to the use of HRV is the time and equipment required to collect this data. Raw HRV data is traditionally collected using an echocardiogram (ECG), then reflected in mathematic calculations either by hand or with software. Several products have been commercialized to streamline this process without a traditional ECG including chest bands, wristbands, and one-lead finger ECG scanners. These have been shown to adequately reflect changes in HRV despite not having the same accuracy as a traditional ECG (Guzik et al., 2018; Perrotta et al., 2017).

The device used in this study was the Elite HRV CorSense monitor. This product is a one-lead ECG that clips on to the subject's index finger. The device is operated via a user-friendly smartphone application available both on iOS and Android phones. It is relatively low cost and calculates the data metrics for HRV within the application giving the user a readout within a minute once a session is completed. This eliminates the need for external software processing. It does not provide a raw rhythm strip like a traditional ECG. The monitor and application are highly mobile, allowing for evaluation away from a medical center.
Other studies that evaluate the adaptive autonomic response to stress have used cardiovascular exercise, resistance training, standing, and exacerbation of the "diver's reflex" as possible stressors (Blake et al., 2016; Gall et al., 2004). The diver's reflex is the transient autonomic response to a cold substance to thermoreceptors located in the face and hands. Prior studies have shown a noticeable change in HRV via RMSSD when comparing the value before, during, and after this reflex using a traditional three-lead ECG (Johnson et al., 2018; Leddy et al., 2017; Leddy et al., 2012). These changes have been appreciable within one minute of exposure, making it an ideal test to monitor the ANS response to a stressor rapidly.

This study is the normative arm of a project originally developed to study athletes before and after concussion. Due to the COVID-19 pandemic only normative data is published here because all athletics were halted at the peak of the pandemic, which prevented sports-related concussions from occurring. Nunan et al. (2010) compiled a systematic review of 44 published articles establishing normative short-interval HRV data in healthy adults. These data were primarily collected with three-lead ECGs or Holter monitoring. The participants in this study also cover the entire age spectrum and have disparate baseline levels of physical activity. The Johnson study discussed above also has sample size $<20$ and focus on females only (Johnson et al., 2018). This study establishes normative data for ambulatory short-interval HRV recording in collegeage men and women with high levels of baseline physical activity using equipment that may be more accessible than a three-lead ECG or Holter monitor in an ambulatory setting. These norms may be useful for athletic trainers, clinicians, or researchers wishing to assess HRV for training or recovery purposes with an accessible and fast method (Dong, 2016).

The goal of this study is to evaluate the utility of the Elite HRV CorSense monitor in detecting a change from baseline using the diver's reflex as a stressor. Our hypothesis is that the diver's reflex produces a sympathetic response from the ANS that can be detected by the RMSSD and LF/HF data in the first minute and then return to baseline as previously shown in other studies (Johnson et al., 2018). If an appreciable difference is detected, this would provide validity for future studies to use the Elite HRV CorSense monitor to rapidly measure HRV rather than a traditional ECG. 


\section{Methods}

\section{Participants}

Sixty-two participants (35 men and 27 women) from Western Michigan University (WMU) football, baseball, softball, basketball, soccer, and volleyball teams were included in this study (Table 1). Demographic information was not collected. Participants were excluded from the study if they were under the age of 18 .
The study protocol was jointly approved by the Institutional Review Boards (IRBs) at both Western Michigan University Homer Stryker M.D. School of Medicine (WMed) and WMU. The target athletes were asked to participate in the study following their presports physical in the WMU Health Center. There was an informed consent process approved by both boards prior to initiation of the protocol described below. All participants who opted-in provided written informed consent and were informed there was no compensation for participation in this study.

Table 1

Participant Demographic Information

\begin{tabular}{lrrrr}
\hline Team & Frequency & Percent & Sex & Frequency \\
\hline Football & 18 & 29.0 & & Percent \\
Men's Baseball & 1 & 1.6 & Male & 27 \\
Men's Basketball & 1 & 1.6 & & 43.6 \\
Men's Soccer & 15 & 24.2 & & \\
Women's Basketball & 1 & 1.6 & Female & 35 \\
Women's Soccer & 18 & 29.0 & & 56.5 \\
Women's Softball & 2 & 3.2 & & \\
Women's Volleyball & 6 & 9.7 & & \\
\hline
\end{tabular}

\section{Study Design}

This was a cross-sectional study, performed in a single session with each participant. For the participants who opted-in, a researcher described the study, an information sheet was provided, and written consent was obtained. The researcher then led the participant through HRV monitoring at rest and then with face cooling (FC) using an Elite HRV CorSense monitor.

\section{Protocol}

The participants were asked to find a comfortable position with eyes closed and the Elite HRV CorSense monitor was placed on the index finger of the participant's choice. A 5-min at-rest reading was recorded, then FC was achieved by placing a pliable $2 \mathrm{~L}$ plastic bag of ice on the forehead, eyes, and cheeks of the participant for a total of three continuous readings recorded at 1 -min intervals. During recording sessions no additional instruction was provided for respiratory pattern.

\section{Elite HRV CorSense Monitor}

The CorSense HRV monitors were purchased from the Elite HRV company for use for this project. Readings were collected through the Elite HRV application for mobile devices. The RMSSD and $\mathrm{LF} / \mathrm{HF}$ data were collected for each reading from the application. Per the Elite HRV website, the platform applies the RMSSD calculation to its recorded R-R intervals via industry standard and defines waves $0.04-0.15 \mathrm{~Hz}$ as LF and waves $0.15-0.40 \mathrm{~Hz}$ as $\mathrm{HF}$.

\section{Limitations}

Demographic information including mental health about the athletes was not collected, and sex was inferred from the athletic team of the participant. There were some technical issues with connecting CorSense HRV monitors to mobile devices, sometimes interfering with data collection times. Much of the detailed information about how Elite HRV specifically calculates RMSSD and LF/HF is proprietary information, and any studies this company has performed verifying the accuracy and precision of their monitors are not public information.

\section{Statistical Analyses}

All data analysis was completed in SAS v9.4 (Cary, NC). Repeated-measure general linear models (GLMs) were used to analyze the relationship of the two outcome measures, RMSSD and LF/HF ratio, to possible predictors including condition (with four levels: at rest, first minute, second minute, and third minute of FC) as well as sex. Sex was tested as a possible predictor in a model that controlled for condition. Due to the positive outliers and right-skew of the LF/HF ratios (Figure 1), the GLM for this outcome measure used the log transformation, 
which better conformed to the normality assumption of GLMs. A Bonferroni-adjusted significance level of 0.0167 was used for the tests for change in HRV from rest at 1,2, and 3 minutes of FC. In order to compare our estimates to the reference values for RMSSD, LF/HF, and Log LF/HF provided by Nunan et al., we calculated our mean and their mean as a percentage of their mean and used the standard deviation they provided in order to calculate a standardized difference (Table 2; Nunan et al., 2010).

\section{Ethical Considerations}

A research ID was generated for all participants in order to deidentify their data. All data were entered into our database using the participant's research
ID. No coaching or training staff had access to the participants' raw or deidentified data.

\section{Results}

\section{RMSSD}

Time after FC initiation was a significant predictor of RMSSD $(p<.0001$, Figure 2$)$. The overall mean $(S D)$ RMSSD at rest was 91 (6.1) ms. RMSSD increased to $138.2(6.1) \mathrm{ms}$ after 1 minute of $\mathrm{FC}$, then dropping to 107.0 (6.1) $\mathrm{ms}$ at 2 minutes of FC, and dropping again to $94.8(6.2) \mathrm{ms}$ at 3 minutes of FC (Table 3). The elevated RMSSD at 1 and 2 minutes of FC were both significant changes from RMSSD at rest, but not at 3 minutes. Controlling for time after FC initiation, sex was not found to be a significant predictor of $\operatorname{RMSSD}(p=.3)$.

\section{Table 2}

Our Mean RMSSD and LF/HF Ratio for the "At Rest" Condition Compared to the Reference Ranges Outlined in Nunan et al. (2010)

\begin{tabular}{|c|c|c|c|c|c|}
\hline Measure & $\begin{array}{l}\text { Our } \\
\text { Mean }\end{array}$ & $\begin{array}{c}\text { Nunan Reference } \\
\text { Mean }\end{array}$ & $\begin{array}{c}\% \\
\text { Difference }\end{array}$ & $\begin{array}{c}\text { Normalized } \\
\text { Difference }\end{array}$ & $\begin{array}{l}\text { Nunan Range } \\
\text { of Means }\end{array}$ \\
\hline RMSSD & 91 & 42 & $117 \%$ & 3.3 & $19-75$ \\
\hline LF/HF Ratio & 1.5 & 2.8 & $-46 \%$ & -0.5 & $1.1-11.6$ \\
\hline
\end{tabular}

Figure 1. Distribution of LF/HF Ratio as a Function of Condition.

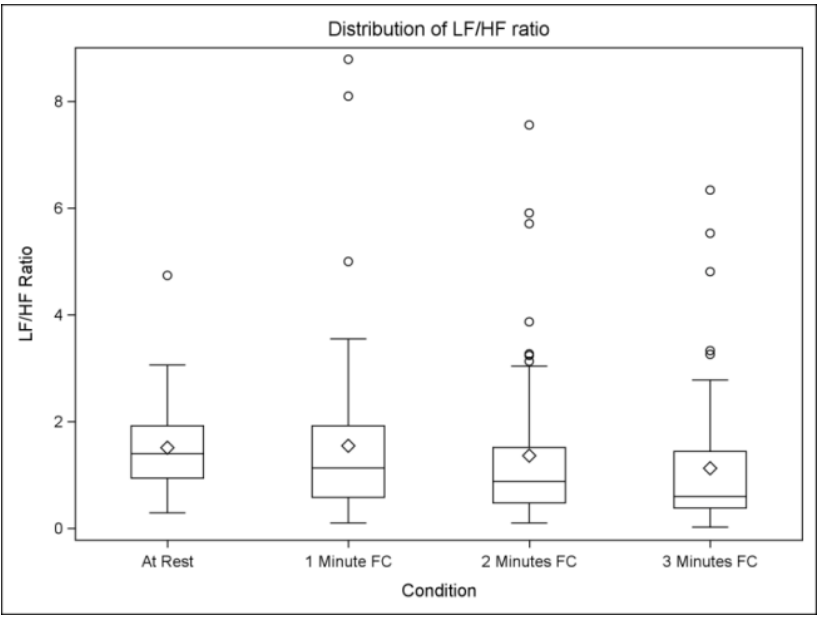

Note. "At rest" refers to the 5-min period of rest prior to face cooling (FC). Each subsequent column refers to the end of the interval at which data was collected. Therefore, 1 minute $\mathrm{FC}$ reflects $\mathrm{HRV}$ during 0 to 1 minutes of $\mathrm{FC}, 2$ minutes FC refers to 1 to 2 minutes, and 3 minutes FC refers to 2 to 3 minutes. Diamond boxes refer to the mean and open circles are outliers. Table 3 lists LF/HF ratio changes and their comparisons to rest with statistical significance.
Figure 2. Distribution of RMSSD as a Function of Condition.

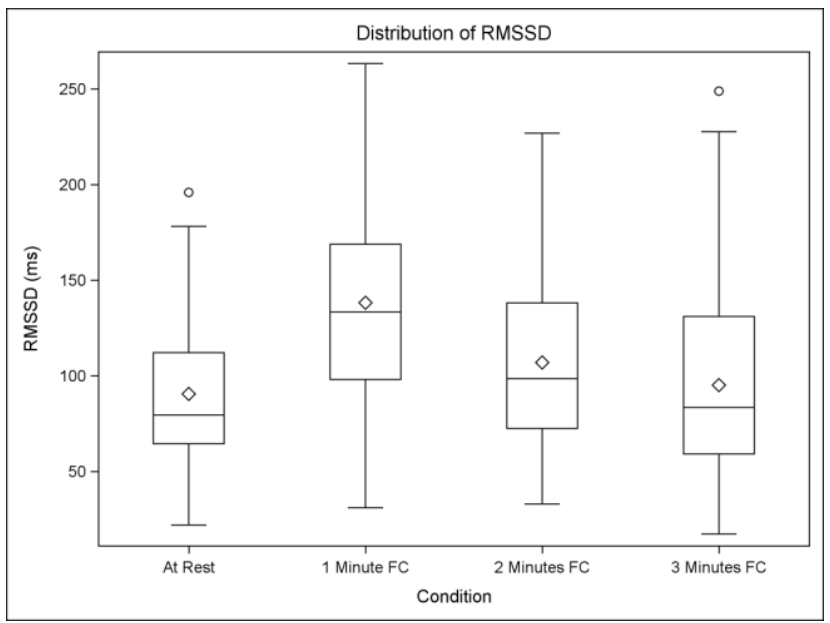

Note. "At rest" refers to the 5-min period of rest prior to face cooling (FC). Each subsequent column refers to the end of the interval at which data was collected. Therefore, 1 minute $\mathrm{FC}$ reflects $\mathrm{HRV}$ during 0 to 1 minutes of $\mathrm{FC}, 2$ minutes FC refers to 1 to 2 minutes, and 3 minutes FC refers to 2 to 3 minutes. Diamond boxes refer to the mean and open circles are outliers. Table 3 lists RMSSD changes and their comparisons to rest with statistical significance. 


\section{Table 3}

Minute of FC Compared to "At Rest" for RMSSD and Raw $L F / H F$

\begin{tabular}{lcccc}
\hline & \multicolumn{2}{c}{ RMSSD } & \multicolumn{2}{c}{ LF/HF } \\
\hline & $\begin{array}{c}\text { Change } \\
\text { from rest } \\
(\mathrm{ms})\end{array}$ & $p$-value & $\begin{array}{c}\text { Change } \\
\text { from rest } \\
(\%)\end{array}$ & $p$-value \\
\hline Minute 1 & 47.7 & $<.0001^{*}$ & -20 & .13 \\
Minute 2 & 16.5 & $.014^{*}$ & -33 & $.007^{*}$ \\
Minute 3 & 4.3 & .52 & -50 & $<.0001^{*}$ \\
\hline${ }^{*} p<.05$ & & & &
\end{tabular}

\section{$\mathrm{LF} / \mathrm{HF}$}

The GLM estimates the mean of the Log LF/HF. Consequently, the LF/HF mean estimates, obtained by exponentiating the GLM estimates, represent geometric means. Time after FC initiation was a significant predictor of Log LF/HF ratio $(p<.0001$, Figure 3 ). The overall mean LF/HF ratio at rest was found to be 1.5 (1.1); mean Log LF/HF ratio at rest was 0.26 (Table 2). LF/HF ratio decreased to 1.04 (1.1) at 1 minute of FC and continued decreasing to 0.88 (1.1) at 2 minutes of $F C$ and 0.65 (1.1) at 3 minutes of FC. The decreased LF/HF ratios at 2 and 3 minutes of $\mathrm{FC}$ were both significant changes from rest, but not at 1 minute. Controlling for time after FC initiation, sex was not found to be a significant predictor of Log LF/HF $(p=.48)$.

Figure 3. Distribution of Log LF/HF by Condition.

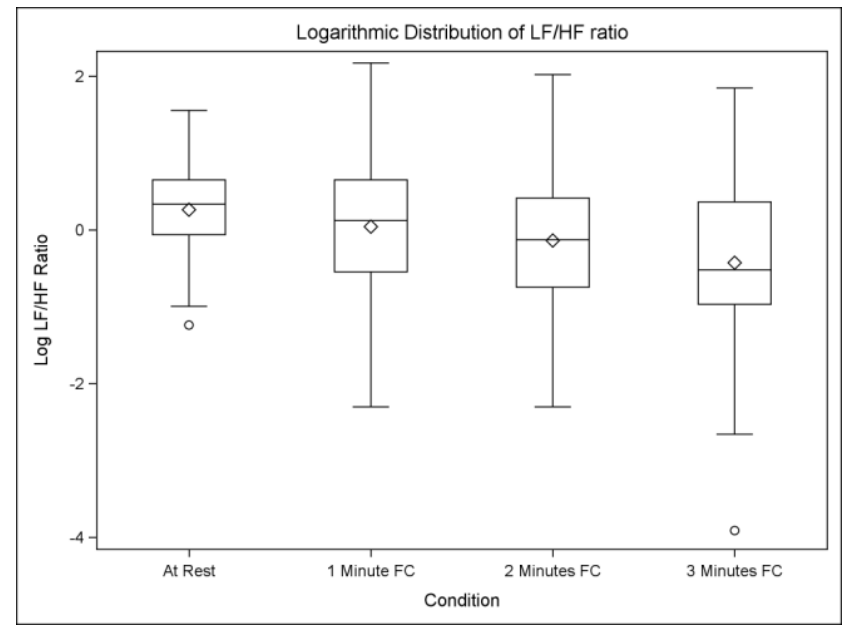

Note. Logarithmic distribution was used due to positive outliers and right skew of the raw LF/HF ratio in Figure 2. Log LF/HF better conformed to the normality assumption in generalized linear models. "At rest" refers to the 5-min period of rest prior to face cooling (FC). Each subsequent column refers to the end of the interval at which data was collected. Therefore, 1 minute FC reflects HRV during 0 to
1 minute of FC, 2 minutes FC refers to 1 to 2 minutes, and 3 minutes FC refers to 2 to 3 minutes. Diamond boxes refer to the mean and open circles are outliers.

\section{Discussion}

The primary findings from this study were that the Elite HRV CorSense monitor was able to detect changes in RMSSD and the LF/HF ratio from rest over 3 min of FC in healthy athletes. RMSSD increased from rest with $\mathrm{FC}$ at $1 \mathrm{~min}$, then fell back to baseline at 2 and $3 \mathrm{~min}$. LF/HF ratio steadily decreased from rest becoming statistically significant beginning at minute 2 of $\mathrm{FC}$. These findings were also consistent across sex; male and female athletes had similar trends in RMSSD and LF/HF ratio over the 3-min FC interval. These data support the use of short intervals (1 and $5 \mathrm{~min}$ ) to monitor RMSSD and LF/HF in college aged athletes using the Elite HRV CorSense monitor.

Our study shows that a baseline can be established for RMSSD and LF/HF ratio using the Elite HRV CorSense monitor and their platform in under 10 minutes. Our methods could be used as a framework for future studies interested in evaluating HRV in the setting of traumatic brain injury (Johnson et al., 2018). The major benefit of a brief protocol is that baselines can be collected for a large group of athletes prior to injury, so each athlete can act as their own control postconcussion; previous studies on HRV with respect to concussion have failed to collect a baseline set of HRV metrics prior to concussion for their subjects.

Having a brief protocol is not without limitations. A 5min recording time was chosen for our at-rest reading time because it has been accepted in the field as adequate for short-term evaluation of RMSSD and the LF/HF ratio (Shaffer \& Ginsberg, 2017). The 1-min recording time with FC was chosen because the diver's reflex is a transient increase in cardiac PNS activity lasting about 1 to 2 minutes, and so a shorter reading was required in order to capture this (Johnson et al., 2018). This means that recordings of variable lengths were compared to each other which is nonideal as longer recordings capture more HRV (Shaffer \& Ginsberg, 2017). Our study demonstrates that despite this complication, a statistical difference between variables was still demonstrated and is a sufficient tool for evaluating HRV in this setting. Of note, LF and HF have established minimum recommended recording times of 2 min and $1 \mathrm{~min}$, respectively, and are less consistent in short reading sessions as a 1min reading could only capture two to nine cycles in 
the LF band, but unfortunately the diver's reflex is not sustained long enough to be captured in a longer reading time (Abhishekh et al., 2013; Berkoff et al., 2007).

An interesting - and counterintuitive-finding of our study was that while RMSSD and LF/HF ratio are both considered markers of $\mathrm{HRV}$, their patterns of change with FC are different. Where the RMSSD was significantly different from rest for the first and second minutes of FC only, LF/HF ratio was significantly different for the second and third minutes, but not for the first. Similar findings were shown in a study by Schipke and Pelzer (2001) who also used the diver's reflex to evaluate HRV through gradual immersion into a pool (Schipke \& Pelzer, 2001). Their study demonstrated that the HF band, which is more reflective of PNS activity, had a more significant change in each stage than the LF band. This led to a decrease in LF/HF ratio in each stage as the participant progressed to full immersion into the pool. We propose that in an analogous way, prolonged FC leads to a gradual increase in HF band through PNS activation, leading to a drop in $\mathrm{LF} / \mathrm{HF}$ after the first minute of FC.

Some confounding factors that have been shown to affect HRV include sex, race, prior medical history, recent alcohol or other substance use, and certain medications (Shaffer \& Ginsberg, 2017). In this study we recruited athletes of both sexes to make our comparisons and found that sex was not a statistically significant confounding variable. We did not, however, separate or screen our subjects for any of the other confounding variables listed above. A comprehensive screen was beyond the scope of our proposal, though important to consider are the temporal factors such as a subject's current medication profile and recent substance use which could transiently affect a subject's baseline. Comparing values following disease to a baseline would ideally be done in similar conditions, and it becomes important to consider how these transient factors could manipulate a subject's baseline or postinjury readings.

Elite HRV and other commercial products have garnered criticism that their platforms and devices may not be as accurate as the three-lead ECG used in many other prior studies (Garabelli et al., 2017; Guzik et al., 2018; Perrotta et al., 2017). When comparing our mean data at rest to the reference range described in Nunan et al. (2010; Table 2), our RMSSD falls outside the range of reported means, but our LF/HF ratio and the log transform are within the range of literature values. On one hand, these data may be reflective of the population differences between the young active adults in our study versus the broad scope of Nunan et al. (2010). On the other hand, it may validate the criticism that Elite HRV is not as accurate at measuring RMSSD as a threelead ECG. Given this discrepancy, the raw values cannot be compared between devices without further research. However, our study does demonstrate that the Elite HRV device is precise enough to detect changes based on condition, and so we would recommend use of Elite HRV when this is the aim. Caution must be taken when comparing HRV metrics between an ECG and a commercial product, and any clinical management of serious conditions affecting mortality should be evaluated with a standard ECG over a 24-hour period rather than the shorter epochs described here. It has not been apparent to our group that the commercial products described in this paper have been explored enough to have utility outside of exercise and sports physiology.

The American Medical Society of Sports Medicine official position statement on concussion from 2019 supports the use of subsymptomatic exercise to expedite recovery from concussion in subjects that can tolerate it (Harmon et al., 2019). This field has begun investigating biologic markers as well as physiologic markers such as HRV (McCrea et al., 2017). Due to the heterogenous nature of concussion, it is likely that HRV would not be a metric to track in all concussions, but instead those with significant autonomic deficits following injury. Having the ability to monitor HRV without an ECG would reduce effort required for researchers, patients, and clinicians to use HRV as a tool in the recovery process.

\section{Conclusion}

The Elite HRV CorSense monitor was able to detect a rise in RMSSD from rest with 1 min of $F C$, and a subsequent fallback to baseline afterwards. This provides a time-efficient and user-friendly framework to measure the adaptive response of the ANS to stressors such as FC, and further research should be done to characterize these changes from baseline in conditions where the ANS is impaired. These data could be used as a reference for researchers and clinicians in evaluating HRV following traumatic brain injury to evaluate the ANS.

\section{Author Disclosure}

No financial disclosures to report. No funds were received from the $\mathrm{NIH}$, Wellcome Trust, or HHMI. Elite HRV CorSense monitors were purchased at 
discount from Elite HRV using funds from the department of orthopedics at WMed. No authors have any conflicts of interest to disclose.

\section{Author Acknowledgements}

To the department of orthopedics at WMed for funding the equipment, the department of athletics at WMU for collaborating, and to the following individuals for significant contributions to data collection: Aditya Mehta BS, Auburn Skakle BS, Gordon Liu BA, Kelsey Suggs MS, Raymond Bayer BS, Robert Welch BS, Spencer Weckwerth BA, Winnie Long BA, Zachary Pearson BS, Rachel Newinsky BS, Rachael Tolsma BS, Maya Giaquinta BS/BA, and Joseph Willner MS.

\section{Trademarked Equipment Used}

Elite HRV CorSense monitor and platform from Elite HRV out of Asheville, NC.

\section{References}

Abhishekh, H. A., Nisarga, P., Kisan, R., Meghana, A., Chandran, S., Raju, T., \& Sathyaprabha, T. N. (2013). Influence of age and gender on autonomic regulation of heart. Journal of Clinical Monitoring and Computing, 27(3), 259-264. https://doi.org/10.1007/s10877-012-9424-3

Berkoff, D. J., Cairns, C. B., Sanchez, L. D., \& Moorman, C. T. (2007). Heart rate variability in elite American track-and-field athletes. The Journal of Strength and Conditioning Research, 21(1), 277-231. https://doi.org/10.1519/00124278200702000-00041

Blake, T. A., McKay, C. D., Meeuwisse, W. H., \& Emery, C. A. (2016). The impact of concussion on cardiac autonomic function: A systematic review. Brain Injury, 30(2), 132-145. https://doi.org/10.3109/02699052.2015.1093659

Collins, M. W., Kontos, A. P., Okonkwo, D. O., Almquist, J., Bailes, J., Barisa, M., Bazarian, J., Bloom, O. J., Brody, D. L., Cantu, R., Cardenas, J., Clugston, J., Cohen, R., Echemendia, R., Elbin, R. J., Ellenbogen, R., Fonseca, J., Gioia, G., Guskiewicz, K., Heyer, R., ... Zafonte, R. (2016). Statements of agreement from the Targeted Evaluation and Active Management (TEAM) approaches to treating concussion meeting held in Pittsburgh, October 15-16, 2015. Neurosurgery, 79(6), 912-929. https://doi.org/10.1227 /NEU.0000000000001447

Dong, J.-G. (2016). The role of heart rate variability in sports physiology (Review). Experimental and Therapeutic Medicine, 11(5), 1531-1536. https://doi.org/10.3892/etm.2016.3104

Esterov, D., \& Greenwald, B. D. (2017). Autonomic dysfunction after mild traumatic brain injury. Brain Sciences, 7(8). https://doi.org/10.3390/brainsci7080100

Gall, B., Parkhouse, W., \& Goodman, D. (2004). Heart rate variability of recently concussed athletes at rest and exercise. Medicine \& Science in Sports \& Exercise, 36(8), 1269-1274. https://doi.org/10.1249/01.MSS.0000135787.73757.4D

Garabelli, P., Stavrakis, S., \& Po, S. (2017). Smartphone-based arrhythmia monitoring. Current Opinion in Cardiology, 32(1), 53-57. https://doi.org/10.1097/HCO.0000000000000350

Guzik, P., Piekos, C., Pierog, O., Fenech, N., Krauze, T., Piskorski, J., \& Wykretowicz, A. (2018). Classic electrocardiogram-based and mobile technology derived approaches to heart rate variability are not equivalent. International Journal of Cardiology, 258, 154-156. https://doi.org/10.1016/j.ijcard.2018.01.056
Harmon, K. G., Clugston, J. R., Dec, K., Hainline, B., Herring, S. A., Kane, S., Kontos, A. P., Leddy, J. J., McCrea, M. A., Poddar, S. K., Putukian, M., Wilson, J. C., \& Roberts, W. O. (2019). American Medical Society for Sports Medicine position statement on concussion in sport. Clinical Journal of Sport Medicine, 29(2), 87-100. https://doi.org/10.1097 /JSM.0000000000000720

Johnson, B. D., O'Leary, M. C., McBryde, M., Sackett, J. R. Schlader, Z. J., \& Leddy, J. J. (2018). Face cooling exposes cardiac parasympathetic and sympathetic dysfunction in recently concussed college athletes. Physiological Reports, 6(9), e13694. https://doi.org/10.14814/phy2.13694

Katz-Leurer, M., Rotem, H., Keren, O., \& Meyer, S. (2010). Heart rate and heart rate variability at rest and during exercise in boys who suffered a severe traumatic brain injury and typically-developed controls. Brain Injury, 24(2), 110-114. https://doi.org/10.3109/02699050903508234

Kleiger, R. E., Stein, P. K., \& Bigger, J. T. (2005). Heart rate variability: Measurement and clinical utility. Annals of Noninvasive Electrocardiology, 10(1), 88-101. https://doi.org /10.1111/j.1542-474X.2005.10101.X

Leddy, J., Baker, J. G., Haider, M. N., Hinds, A., \& Willer, B. (2017). A physiological approach to prolonged recovery from sport-related concussion. Journal of Athletic Training, 52(3), 299-308. https://doi.org/10.4085/1062-6050-51.11.08

Leddy, J. J., Sandhu, H., Sodhi, V., Baker, J. G., \& Willer, B. (2012). Rehabilitation of concussion and post-concussion syndrome. Sports Health, 4(2), 147-154. https://doi.org $/ 10.1177 / 1941738111433673$

McCrea, M., Meier, T., Huber, D., Ptito, A., Bigler, E., Debert, C. T., Manley, G., Menon, D., Chen, J.-K., Wall, R., Schneider, K. J., \& McAllister, T. (2017). Role of advanced neuroimaging, fluid biomarkers and genetic testing in the assessment of sport-related concussion: A systematic review. British Journal of Sports Medicine, 51, 919-929. https://doi.org/10.1136 /bjsports-2016-097447

Miranda, N. A., Boris, J. R., Kouvel, K. M., \& Stiles, L. (2018). Activity and exercise intolerance after concussion: Identification and management of postural orthostatic tachycardia syndrome. Journal of Neurologic Physical Therapy, 42(3), 163-171. https://doi.org/10.1097 /NPT.0000000000000231

Nunan, D., Sandercock, G. R. H., \& Brodie, D. A. (2010). A quantitative systematic review of normal values for short-term heart rate variability in healthy adults. Pacing and Clinical Electrophysiology, 33(11), 1407-1417. https://doi.org/10.1111 /j.1540-8159.2010.02841.x

Patricios, J. S., Ardern, C. L., Hislop, M. D., Aubry, M., Bloomfield, P., Broderick, C., Clifton, P., Echemendia, R. J., Ellenbogen, R. G., Falvey, E. C., Fuller, G. W., Grand, J., Hack, D., Harcourt, P. R., Hughes, D., McGuirk, N., Meeuwisse, W., Miller, J., Parsons, J. T., Richiger, S., ... Raftery, M. (2018). Implementation of the 2017 Berlin Concussion in Sport Group Consensus Statement in contact and collision sports: A joint position statement from 11 national and international sports organisations. British Journal of Sports Medicine, 52(10), 635-641. https://doi.org /10.1136/bjsports-2018-099079

Perrotta, A. S., Jeklin, A. T., Hives, B. A., Meanwell, L. E., \& Warburton, D. E. R. (2017). Validity of the elite HRV smartphone application for examining heart rate variability in a field-based setting. The Journal of Strength and Conditioning Research, 31(8), 2296-2302. https://doi.org /10.1519/JSC.0000000000001841

Schipke, J. D., \& Pelzer, M. (2001). Effect of immersion, submersion, and scuba diving on heart rate variability. British Journal of Sports Medicine, 35, 174-180. https://doi.org /10.1136/bjsm.35.3.174

Senthinathan, A., Mainwaring, L. M., \& Hutchison, M. (2017). Heart rate variability of athletes across concussion recovery milestones: A preliminary study. Clinical Journal of Sport 
Medicine, 27(3), 288-295. https://doi.org/10.1097 JSSM.0000000000000337

Shaffer, F., \& Ginsberg, J. P. (2017). An overview of heart rate variability metrics and norms. Frontiers in Public Health, 5, 258. https://doi.org/10.3389/fpubh.2017.00258

Stein, P. K., Domitrovich, P. P., Hui, N., Rautaharju, P., \& Gottdiener, J. (2005). Sometimes higher heart rate variability is not better heart rate variability: Results of graphical and nonlinear analyses. Journal of Cardiovascular Electrophysiology, 16(9), 954-959. https://doi.org/10.1111 /j.1540-8167.2005.40788.x
Vistisen, S. T., Hansen, T. K., Jensen, J., Nielsen, J. F., \& Fleischer, J. (2014). Heart rate variability in neurorehabilitation patients with severe acquired brain injury. Brain Injury, 28(2), 196-202. https://doi.org/10.3109 /02699052.2013.860477

Received: February 11, 2021

Accepted: April 8, 2021

Published: June 30, 2021 